\title{
Intra-ventricular blood flow simulation with patient specific geometry
}

\author{
Q. Long ${ }^{1}$, R. Merrifield ${ }^{2}$, X. Y. Xu ${ }^{1}$, P. J. Kilner ${ }^{3}$, D.N. Firmin ${ }^{3}$, G. Z. Yang ${ }^{2}$ \\ ${ }^{1}$ Dept. of Chem. Eng. \& Chem. Tech., Imperial College, London, UK q.long@ic.ac.uk \\ ${ }^{2}$ Dept. of Computing, Imperial College, London, UK. \\ ${ }^{3}$ Cardiovascular MR Unit, Royal Brompton Hospital, Imperial College, London, UK
}

\begin{abstract}
This study demonstrates a subject specific approach for simulating blood flow within a left ventricle (LV) by a combined analysis of computational fluid dynamics (CFD) and magnetic resonance imaging (MRI). Time dependent LV morphology and wall movements are measured by 3D multi-slice MRI. The information is then used to build numerical meshes for CFD simulation which provides detailed 3D LV velocity and pressure distributions in the $L V$ cavity. Issues related to mesh generation and the prescription of valvular boundary conditions are also discussed. The predicted flow patterns are compared to that of the published MR measurements, demonstrating the effectiveness of the proposed approach.

Keywords - CFD; MRI; left ventricle; blood flow
\end{abstract}

\section{INTRODUCTION}

Heart disease is one of the biggest killer and debilitating factor in the west world. Thus far, investigators have focused on abnormalities of the LV function to explore signs and symptoms of heart disease[1-3]. LV function of the heart involves many interrelated events both in systole and diastole. Numerous factors determine how the ventricle is filled with blood during diastole and how blood is ejected during systole. Each of these factors, including ventricular relaxation, diastolic suction, ventricular contraction, pericardial restraint and ventricular interaction, is interrelated to the others in a complex sequence of events[4-6]. Because blood flow in the LV is determined by a combination of these factors, a detailed investigation of intraventricular flow patterns can provide practical insight that may facilitate the diagnosis and treatment of $\mathrm{LV}$ dysfunction.

CFD techniques have been used to investigate LV flow based on idealised models for many years and is undergoing significant improvements recently. Combining CFD with non-invasive imaging techniques has been proven to be an effective means of studying complex cardiovascular dynamics, being able to provide detailed haemodynamic information that is unobtainable by using direct measurement techniques. Depending on how the ventricular wall is treated in the model, CFD simulations of intra-ventricular flow can

- This work is sponsored by the British Heart Foundation (FS/2001002) be classified into two categories. The first one treats dynamic interactions of blood and the ventricular wall, so that the wall moves in response to fluid forces and changes in tissue characteristics by allowing the wall properties to be time-varying[7-11]. The second category treats the ventricular wall as a moving boundary and blood flow was driven by the prescribed wall movement[12-14]. Because of the difficulty in obtaining time-dependent wall properties in vivo, simulations on the first category were mostly performed on idealised models with simplified geometries. The latest advancement in MRI permits accurate measurement of ventricular anatomy throughout the cardiac cycle, allowing simulations based on the second approach to be more realistic by using MR measured subject-specific $\mathrm{LV}$ wall movement as simulation geometry boundary.

The purpose of this paper is to describe a study of simulating intra-ventricular flow by the combination of CFD and MRI based on the moving boundary approach. In the study, anatomically realistic LV wall motions were measured by 3D multi-slice MRI. With the prescribed LV wall movement as well as boundary conditions defined on the aortic (for systolic phase) and mitral (for diastolic phase) valve planes, flow simulations were performed on the whole LV cavity throughout cardiac cycle.

\section{METHOLDS}

A healthy volunteer with no history of heart disease was chosen for this study. The study includes the following steps: (1) MRI of the left ventricle to acquire time dependent ventricular geometry; (2) Image processing to construct a deformable LV model; (3) Mesh generation, interpolation and CFD simulations.

\section{A. MR data acquisition and image processing}

Imaging was performed using a Siemens Sonata $1.5 \mathrm{~T}$ MR system. A multi-slice cine True-FISP imaging sequence was used to acquire 7 short axis slices at 16 phases providing complete spatial and temporal coverage of the LV. Breath-holding was used in MRI so as to minimize registration errors caused by respiratory movement. Segmentation and reconstruction of the LV structure was performed using proprietary software. This allowed the capture of detailed spatial and temporal properties of the endocardial border from reformatted images 
representing short axis, long axis and M-Mode acquisitions.

\section{$B$ Structured mesh generation and CFD simulation}

For each time phase, structured grids consisting of hexahedrall cells were generated in the LV cavity. Details of mesh generation can be found in[15]. The final adopted model contained 54,230 nodes and 41,000 hexahedrall cells. A total of 16 meshes were generated from the original image data in one cycle. Cubic splines were employed to interpolate the 16 meshes into 49 meshes to provide a fine time step for CFD simulations.

The Navier-Stokes equations for 3D time-dependent laminar flow with moving walls were solved using a finite-volume based CFD solver CFX4 (CFX international, AEA technology, Harwell). The blood was treated as an incompressible Newtonian fluid with a constant viscosity of $0.004 \mathrm{Kg} /(\mathrm{ms})$. The simulation started from the beginning of systole with zero pressure defined at the aortic valve plane while the mitral valve plane was treated as no-slip wall. When it reached the beginning of diastole the aortic valve was closed (treated as wall) and the mitral valve plane was allowed to open (treated as a combination of pressure and velocity boundaries) with a 'plug' velocity profile. The simulation was repeated for four cycles to reach a periodic solution and results obtained in the fourth cycle are presented here.

Tests on mesh sensitivity and time-step size were performed by comparing results obtained with two different mesh densities (61,992 cells vs. 41,000 cells) but the same number of time steps (Test 1 ), as well as those with the same mesh density $(41,000$ cells) but two different time-steps ( 49 vs. 80 per cycle, Test 2 ). Numerical differences in predicted velocities were found to be $4 \%$ for Test 1 and $2.5 \%$ for Test 2 at chosen test points, the mesh consisting of 41,000 cells and a time-step of 49 per cycle were adopted in this study. 3D graphic package EnSight was used as CFD post-processor to visualize the CFD results.

\section{RESULTS}

A Geometrical reconstruction

Two planes (A-B and C-D) were defined to demonstrate the predicted velocity vectors as shown in Figure 1 (a). Plane A-B is approximately anteroposteriorly orientated long-axis plane, aligned with both inflow and outflow valves. Plane C-D is approximately inferior-superiorly orientated ling-axis plane orthogonal to the plane A-B.

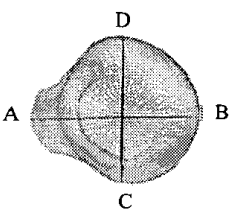

(a)

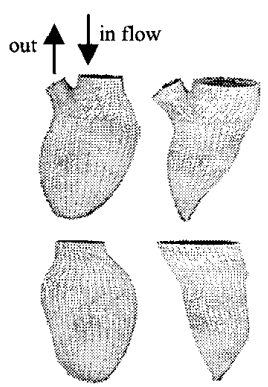

(b) (c)
Figure 1. Reconstructed 3D LV model geometry. (a) locations of chosen plane for presenting velocity results; (b) surface mesh of $\mathrm{LV}$ at beginning of systole , (c) at beginning of diastole. Upper panel shows A-B plane view and lower panel shows $C$-D plane view;

3D surface of the LV model is presented in Figure 1 from two different viewing angles (A-B view at upper panel and C-D view at lower Panel) at the beginning of

systole (b) and diastole (c). The structured mesh for the LV model is also shown in Figure 1. Significant shape and volume changes can be evidenced during the cardiac cycle. The movements along the $\mathrm{B}$ side wall (corresponding to the free wall) and the $D$ side wall were larger than $A$ and $C$ wall. The inflow track area $A_{t}$ changed during the cycle. It should be noted here that neither mitral and aortic valve leaflets had been modelled in the study, nor had the exact valve ring locations due to lack of information. Instead, the boundary planes were set at about $5 \mathrm{~mm}$ above the ventricle base which were probably inside the left atrium and ascending aorta. For the subject, the ratio of systole/diastole is about 0.47 . (or $\mathrm{t} / \mathrm{tp}=0.47$ for the end of systole).

\section{B. Calculated flow patterns}

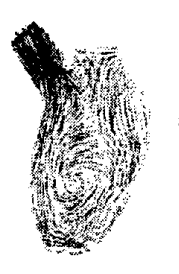

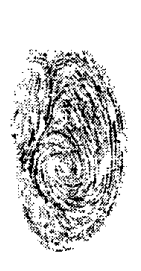

C-D

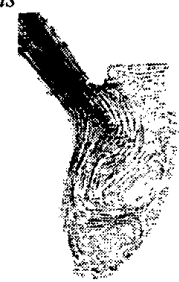

A-B

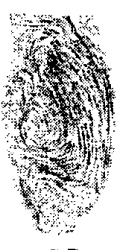

C-D (a)

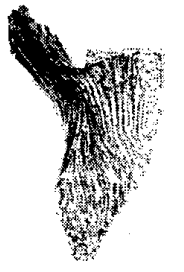

(b)

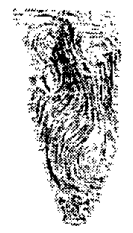

Figure 2. Velocity vectors on the A-B and C-D planes at (a) early systole, $t / t \mathrm{p}=0.04$, (b) mid-systole, $t / \mathrm{tp}=0.16$, (c) late systole, $t / \mathrm{tp}=0.30$ 
Figure 2 (a), (b) and (c) show velocity vectors on planes A-B (left panel) and C-D (right panel) at three time phases in systole.

It can be found that the inherited vortices from diastole dominate the flow patterns at the beginning of systole (in Figure 2(a), $t / t \mathrm{p}=0.04)$ with clockwise and anticlockwise vortices in the A-B and C-B planes respectively. Similar flow patterns can still be seen in (b) and fade away gradually by further ventricular contraction. Because of these, the particles flow along the A-D side of LV to be pumped out to ascending aorta. This may be observed even in late stages of systole as in Figure 5(c). It is also interesting to note that the locations of the vortices' centre do not move very much from (a) to (b).

Predicted velocity vectors on the A-B and C-D planes in diastole were shown in Figure 3. The main flow feature found for the diastole phase was that blood flowed into LV cavity via mitral valve and formed a jet towards LV apex. Vortex formed at the second half of diastole with clockwise and anti-clockwise flow in planes A-B and C-D respectively. In early diastole (Fig. 3(a) $t / t \mathrm{p}=0.56$ ), the inflow jet is wide and almost occupied the whole LV region. Going into the second half of diastole $(t / t p=0.70)$ the jet was getting narrow with a vortex formed at the A-D side of the jet as shown in Figure 3(b). With further relaxation of $L V$, mitral valve plane became smaller as well as the jet flow region, another vortex could be seen on the B-C side of the wall during these phases. At the end of diastole (Figure $3(\mathrm{c}) \mathrm{t} / \mathrm{tp}=0.97$ ), inflow rate was decreased significantly, the vortex which was formed in figure 3(b) is now moved towards centre of $\mathrm{LV}$ cavity which would remain until mid-systole of next cycle.

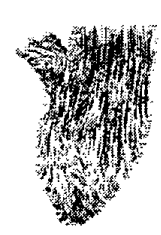

$A \cdot B$

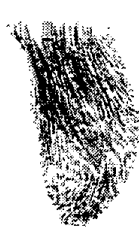

C-D

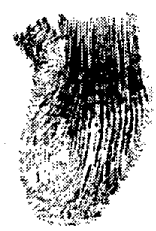

A-B (a)

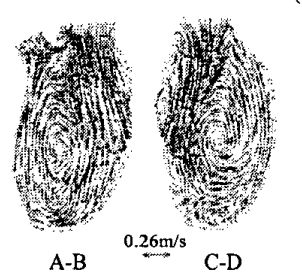

(c)

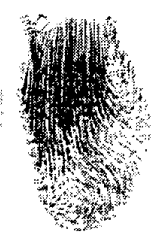

C-D (b)

Figure 3. Velocity vectors on the A-B and C-D planes at (a) early diastole, $\mathrm{t} / \mathrm{t} \mathrm{p}=0.56$, (b) mid-diastole, $\mathrm{t} / \mathrm{t}=0.70$, (c) late diastole, $\mathrm{t} / \mathrm{t}=0.97$.

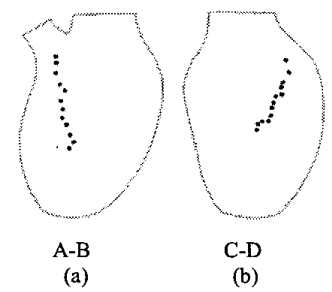

Figure 4. Vortex track during the second half of diastole. Each point corresponding one time phase which starts at $\mathrm{t} / \mathrm{t}=0.70$ from the point near $L V$ base and ends at end diastole at $L V$ cavity centre.

\section{$C$ Vortex formation}

From Figure 3(b) and (c), it was found that the vortex formed at the A-D side of jet moved towards LV cavity centre from mid-diastole to the end-diastole. Figure 4 presented the track of the vortex centre movements at two viewing angles. Each point presented the vortex centre location at a time phase. From left panel (A-B plane), it showed that the vortex centre remained near A side of wall for a relatively long time and then turned to the LV centre (the last four points). At C-D plane, vortex moved to the LV centre along an almost straight line. Considering the fact that the vortex is always on the side of the inflow jet, the width of the jet decreased quicker in C-D plane than those in A-B plane.

\section{DISCUSSION}

Numerical simulation of LV flow on geometrical realistic model has rarely been undertaken because of the difficulties of acquiring time dependent LV morphology and wall movements in vivo as well as generating numerical meshes for the CFD simulation. With the latest advancement in MRI, we are now able to obtain the geometry information in good accuracy. The present study shows that the combination of CFD and MRI is capable to provide detailed LV flow patterns on patient specific bases.

However, computational modelling of LV flow on this manner is subject to a number of potential errors. Uncertainties can come from MRI, LV model reconstruction as well as CFD simulation. Proper selection of inflow boundary condition on the mitral valve plane during diastole is one of the most important factors affecting the accuracy of flow predictions. Ideally, MR measured velocity profiles of trans-mitral flow should be defined as inflow boundary condition on mitral valve plane. However, in our model, inflow volumetric inflow rate via matial valve was defined only by the LV volume change at each time phase. To avoid over constraining the inflow rate and satisfy global mass conservation, a hybrid 
boundary condition type was used in the study. In doing so, the inflow boundary plane was divided in two part. On one part, which occupied main area of the plane, velocity boundary condition was used in which specific velocity values were defined on each cell. On the other part, pressure boundary condition was defined to achieve global mass conservation for CFD calculation. No MR measured trans-mitral velocity profile available for the studied subject, according to the published measurement results[16,17], as an approximation, 'Plug' flow profile was used on inflow plane for every time step.

Although no direct comparison of the predicted flow patterns with MR measurements have been made in the study, the main flow features during diastole were qualitatively similar to the reported[18]. The main inflow entrance the ventricle towards the apex with slight skew to the B-C wall and vortices formed at both side of the inflow jet with the A side one is larger than the B side. Quantitative validation results will be presented in the conference.

\section{CONCLUSIONS}

The combination of MRI and CFD is able to predict LV blood flow with faithful mesh generation and the appropriately selected boundary conditions. The main flow features predicted by the method are similar to the reported MR velocity mapping results. However, further validations are necessary should the protocol be applied on a large scale. More detailed validations will be presented on the conference.

\section{ACKNOWLEDGEMENT}

This study is sponsored by the British Heart Foundation (FS/2001002)

\section{REFERENCES}

1. M. Packer. Abnormalities of diastolic function as a potential cause of exercise intolerance in chronic heart failure. Circulation 1990; 81 Suppl III:III 7886.

2. I. Mirsky, A. Pasipoularides. Clinical assessment of diastolic function. Prog Cardiovasc Dis 1990; 32: 291-318.

3. W.H. Gaasch, H.J. Levine, M.A. Quinones, J.K. Alexander. Left ventricular compliance: mechanisms and clinical implications. Am J Cardiol 1976; 38: 645-53

4. D.L. Brutsaert, S.U. Sys. Relaxation and diastole of the heart. Physiol Rev 1989; 69: 1228-315.

5. J.C. Gilbert, S.A. Glantz. Determinants of left ventricular filling and of the diastolic pressurevolume relation. Cir Res 1989; 64: 827-52.

6. W.C. Little, T.R. Downes. Clinical evaluation of left ventricular diastolic performance. Prog Cardiovasc Dis 1990; 32: 273-90
7. C.S. Peskin, D.M. McQueen. Modelling prosthetic heart velves for numerical analysis of blood flow in the heart. J Computational Physics. 1980; 37: 113-32.

8. D.M. McQueen, C.S. Peskin. Computer-assisted design of butterfly bileaflet valves for the mitral position. Scand J Thor Cardiovasc Surg. 1985; 19: $139-48$.

9. B. Chahboune, J.M. Crolet. Numerical simulation of the blood-wall interaction in the human left ventricle. European Physical J Applied Physics. 1998; 2: 291-97.

10. C.S. Peskin, D.M. McQueen. Cardiac fluiddynamics, Crit Rev Biomed Eng 1992; 20: 451-59.

11. P. Verdonck, J. Vierendeels, K. Riemslagh, E. Dick. Left-ventricular pressure gradients: a computer-model simulation. Medical \& Biological Engineering \& Computing, 1999; 37: 511-15.

12. E. Gonzalez, R.T. Schoephoerster. A simulation of three-dimensional systolic flow dynamics in a spherical ventricle: effects of abnormal wall motion. Annals of Biomed Eng 1996; 24: 48-57.

13. T.W. Taylor, T. Yamaguchi. Realistic threedimensional left ventricular ejection determined from computational fluid dynamics. Med End Phys 1995; 17: 602-08.

14. N.R. Saber, A.D. Gosman, N.B. Wood, P.J. Kilner, C. Charrier and D.N. Firmin, Computational flow modelling of the left ventricle based on in vivo MRI data - initial experience, Annals Biomed Eng 2001,29:275-283

15. Q. Long, X.Y. Xu, M.W. Collins, M. Bourne and T.M. Griffith, "Magnetic resonance image processing and structured grid generation of a human abdominal bifurcation". Computer Methods and Programs in Biomedicine, 1998, 56:249-259

16. S. Fujimoto, R.H. Mohiaddin, K.H. Parker, D.G. Gibson, Magnetic resonance velocity mapping of normal human transmitral velocity profiles, Heart and Vessels, 1995, 10:236-240.

17. Y.H. Kim, P.G. Walker, E.M. Pedersen, J.K Poulsen, S. Oyre, K. Houlind, A.P. Youganathan. Left ventricular blood flow patterns in normal subjects: a quantitative analysis by $3 \mathrm{D}$ magnetic resonance velocity mapping. J Am Coll Cardiol, 1995, 26:224-238

18. P.J. Kilner, G.Z. Yang, A.J. Wilkes, R.H. Mohiaddin, D.N. Firmin, M.H. Yacoub, Asymmetric redirection of flow through the heart, Nature 2000, 404:759-761 